\title{
Degradación del posicionamiento de precisión originada por irregularidades de plasma ecuatorial
}

\author{
IZARrA RodríGuez BILBAO ${ }^{1}$, BEATRIZ MORENO MONGE ${ }^{2}$, Gracia \\ RODRÍGUEZ-CADEROT ${ }^{1,5}$, MigUEL HERRAIZ SARACHAGA ${ }^{1,3}$, \\ SANDRO MARIA RADICELLA ${ }^{4}$ \\ ${ }^{1}$ Dpto. de Física de la Tierra Astronomía y Astrofísica I. Universidad Complutense, Madrid, España. \\ irbilbao@ucm.es \\ ${ }^{2}$ Centro de Innovación de Infraestructuras Inteligentes (Ci3), Guadalajara, España. \\ ${ }^{3}$ Instituto de Geociencias, (UCM, CSIC), Madrid, España. \\ ${ }^{4}$ The Abdus Salam International Center for Theoretical Physics, T/ICT4D Laboratory, Trieste, Italia. \\ ${ }^{5}$ Instituto de Matemática Interdisciplinar. UCM.
}

Recibido: 20/05/2014

Aceptado: 23/09/2014

\section{Resumen}

La variabilidad ionosférica constituye una de las principales amenazas sobre las técnicas basadas en GNSS, tanto en la medida de parámetros ionosféricos como en el posicionamiento de precisión. En este trabajo se muestra el efecto de las irregularidades de plasma ecuatorial sobre la pérdida de señal y su influencia en el posicionamiento puntual de precisión. Los resultados ponen de manifiesto la importancia que tiene la capacidad del receptor para no perder la señal proveniente de los satélites GNSS en la precisión del posicionamiento.

Palabras clave: ionosfera, GNSS, PPP, irregularidades ionosféricas.

\section{Precise positioning accuracy degradation caused by equatorial plasma irregularities}

\begin{abstract}
Ionospheric variability is one of the main threats to GNSS-based techniques as ionospheric parameters measurement and precise positioning. In this work the effect of equatorial plasma irregularities on signal loss and its influence on the precise point positioning accuracy are shown. These results highlight the importance of the receiver capacity to deal with signal loss and its effects on positioning accuracy.
\end{abstract}

Key words: ionosphere, GNSS, PPP, ionospheric plasma irregularities.

Sumario: Introducción 1. Datos y Procedimiento. 2. Resultados. 3. Conclusiones. Agradecimientos. Referencias Bibliográficas.

\section{Referencia normalizada}

Rodríguez-Bilbao, I., Moreno, B., Rodríguez-Caderot, G., Herraiz, M., Radicella, S.M. (2014). Degradación del posicionamiento de precisión originada por irregularidades de plasma ecuatorial. Física de la Tierra, Vol. 26, 89-100. 


\section{Introducción}

La ionosfera de bajas latitudes se caracteriza por la presencia de la Anomalía de Ionización Ecuatorial: dos picos de densidad electrónica a unos $15^{\circ}$ Norte y Sur del ecuador magnético separados por un valle de menor densidad electrónica sobre el ecuador magnético. Esta singularidad en la ionosfera terrestre tiene su origen en la deriva vertical del plasma ecuatorial desde la región $\mathrm{E}$ a la $\mathrm{F}$ a causa de la perpendicularidad entre el campo eléctrico zonal dirigido hacia el Este durante el día (y hacia el Oeste durante la noche) y el campo magnético terrestre, que en bajas latitudes es prácticamente horizontal y está dirigido hacia el polo Sur magnético (situado en el hemisferio Norte). Los iones y electrones se desplazan a lo largo de las líneas de campo para descender después bajo la influencia de los gradientes de presión y gravedad y dar lugar a los dos máximos de densidad electrónica mencionados (Risbeth, 2000). Poco antes de la puesta del Sol, el campo eléctrico mencionado se acentúa (lo que se conoce como "aumento pre-inversión", o PRE, del inglés prereversal enhancement) y como consecuencia la Anomalía se intensifica favoreciendo la formación de irregularidades de plasma (Abdu, 2001). Los distintos fenómenos de irregularidades en la densidad electrónica del plasma que se dan en la capa $\mathrm{F}$ ecuatorial con escalas que van desde decenas de centímetro hasta centenas de kilómetros, se agrupan en el fenómeno que se conoce como "dispersión de la capa F ecuatorial" (ESF, del inglés Equatorial Spread F). Estas irregularidades se dan principalmente durante la noche y evolucionan a partir de perturbaciones de plasma en la parte baja de la región F a través del mecanismo de Rayleigh-Taylor (Sultan, 1996). Dichas perturbaciones crecen y ascienden hacia la parte alta de la región $F$, dando lugar a estructuras con una densidad menor que la del entorno, conocidas como burbujas de plasma ecuatorial. Las burbujas se caracterizan por un fuerte descenso en el contenido total de electrones (TEC, del inglés Total Electron Content) donde pueden coexistir irregularidades de menor escala creando fuertes gradientes de densidad electrónica (Basu et al., 1978). La generación de irregularidades en la densidad electrónica de la ionosfera ecuatorial está estrechamente ligada al PRE (Li et al., 2007; Whalen 2002) y por lo tanto, al igual que éste, el ESF también experimenta variabilidad debida a la localización longitudinal, la estación del año y la actividad solar (Tsunoda, 1985; Gentile et al., 2011).

La ionosfera es un medio dispersivo en cuanto a la propagación de señales GNSS (Global Navigation Satellite System) se refiere, por lo que el retardo (adelanto) producido sobre la velocidad de grupo (fase) depende, por una parte, del TEC que ha sido atravesado por la señal y, por otra, de su frecuencia. La posición de un receptor GNSS de doble frecuencia puede determinarse mediante la técnica conocida como "posicionamiento puntual de precisión" (PPP). Se trata de un método de post-procesado que utiliza observaciones en doble frecuencia de una sola estación y productos precisos de los satélites donde el efecto de la ionosfera se puede eliminar en primer orden mediante una combinación lineal de los observables que se conoce como combinación libre de ionosfera, y así obtener un posicionamiento de precisión 
del orden de centímetros en modo estático y de decímetros en modo cinemático (Bisnath and Gao, 2007). Sin embargo, las irregularidades en la densidad electrónica causan inhomogeneidades en el índice de refracción del medio y por lo tanto en la refractividad de las señales de radiofrecuencia que las atraviesan, por lo que bajo fuertes fluctuaciones de TEC pueden observarse errores significativos en el posicionamiento de precisión (Moreno et al., 2011).

En este trabajo se presenta el efecto de las irregularidades del plasma ionosférico, identificadas mediante la variación temporal del TEC (ROT, del inglés Rate of change of TEC), en la pérdida de señal y el PPP. Al mismo tiempo, se muestra la influencia que tiene el tipo de receptor en la capacidad de hacer frente a los problemas derivados de las fluctuaciones rápidas de contenido electrónico y cómo los nuevos receptores y productos proporcionados por el IGS han producido una clara mejora del posicionamiento de precisión.

\section{Datos y Procedimiento}

En este estudio se ha trabajado con datos de los años 2001, 2004, 2008 y 2011 de la estación permanente KOUR (Kourou, Guayana Francesa) integrada en el IGS (International GNSS Service). Se han seleccionado dichos años ya que se encuentran bajo distintas condiciones de actividad solar (véase tabla 1) a lo largo de los cuales el receptor ha sido reemplazado en varias ocasiones (véase tabla 2).

Tabla. 1. Características de la estación KOUR (modelo del campo geomagnético IGRF-11 para 2008) y número de archivos de cada año utilizados en el estudio, así como el promedio anual de manchas solares. (*http://wdc.kugi.kyoto-u.ac.jp, **http://sidc.oma.be/index.php)

\begin{tabular}{|c|c|c|c|c|c|c|c|c|c|}
\hline \multicolumn{2}{|c|}{$\begin{array}{c}\text { Coordenadas } \\
\text { Geográficas }\end{array}$} & \multicolumn{2}{|c|}{$\begin{array}{c}\text { Coordenadas } \\
\text { Geomagnéticas } \\
*\end{array}$} & \multicolumn{2}{|c|}{$\begin{array}{c}\text { Componentes } \\
\text { del } \\
\text { Campo } \\
\text { Geomagnético* }\end{array}$} & \multicolumn{4}{|c|}{ Número de archivos } \\
\hline $\begin{array}{l}\text { Lat } \\
\left({ }^{\circ} N\right)\end{array}$ & $\begin{array}{l}\text { Lon } \\
\left({ }^{o} E\right)\end{array}$ & $\begin{array}{l}\text { Lat. } \\
\left({ }^{o} N\right)\end{array}$ & $\begin{array}{l}\text { Lon. } \\
\left({ }^{o} E\right)\end{array}$ & $D\left({ }^{o} E\right)$ & $I\left(\left(^{o}\right)\right.$ & 2001 & 2004 & 2008 & 2011 \\
\hline 5.25 & 307.10 & 14.73 & 19.74 & -17.89 & 18.05 & 269 & 351 & 350 & 363 \\
\hline \multicolumn{6}{|c|}{ Promedio anual de manchas solares $* *$} & 111.0 & 40.4 & 2.9 & 55.7 \\
\hline
\end{tabular}

El primer paso del trabajo ha consistido en el cálculo del retardo ionosférico para cada par satélite-receptor mediante la técnica descrita en De Lacy et al. (2005, 2008) a partir de los datos de código y fase registrados en ambas frecuencias GPS L1 y L2 (f1 $=1575.42 \mathrm{MHz}$ y f2 $=1227.60 \mathrm{MHz}$ ) y así determinar el TEC oblicuo o sTEC (del inglés slant TEC) con el mismo procedimiento llevado a cabo por Moreno et al. (2011). Se ha utilizado un modelo ionosférico de capa simple (Schaer, 1997) a 350 $\mathrm{km}$ de altura para convertir las observaciones de sTEC a TEC vertical equivalente 
(vTEC) en cada punto subionosférico (IPP, Ionospheric Pierce Point). Típicamente se suele considerar un valor de entre $300 \mathrm{~km}$ y $600 \mathrm{~km}$. La diferencia entre considerar uno u otro produce variaciones en la función de mapeo que pueden alcanzar hasta un $11 \%$ a bajas elevaciones, pero que se reduce por debajo de un $2 \%$ si se consideran elevaciones superiores a $45^{\circ}$. Sin embargo los fuertes gradientes espaciales pueden introducir mayores errores en dicha conversión (Nava et al., 2007).

En un segundo paso se ha procedido al cálculo del ROT a partir de la primera derivada temporal del vTEC. De este modo hemos obtenido las fluctuaciones del TEC en intervalos de 30s (velocidad de muestreo de los ficheros utilizados). Se han analizado los primeros 90 días de 2001 para calcular cual es la diferencia que existe en el valor del ROT al considerar una altitud de la capa simple a $300 \mathrm{~km}$ o a $600 \mathrm{~km}$. Se ha determinado que esta desviación alcanza 0.02TECU/30s en el $97 \%$ de los casos siempre que se consideren elevaciones superiores a $45^{\circ}$. Este porcentaje cae hasta el $65 \%$ al considerar un umbral de $30^{\circ}$ en lugar de $45^{\circ}$. Se ha escogido un valor umbral en la elevación de los satélites de $45^{\circ}$ ya que disminuye el error producido por la función de mapeo y al mismo tiempo evita los problemas derivados de obstáculos y de camino múltiple de la señal.

Para determinar un valor umbral en el ROT a partir del cual poder considerar que se están observando irregularidades ionosféricas, se han analizado los valores de ROT de satélites con elevación superior a $45^{\circ}$, de 10 días de un año con alta actividad solar en el que no se observan fuertes perturbaciones ionosféricas (del 31 de mayo al 10 de junio del 2001). Se ha determinado que el valor atípico extremo obtenido a partir del rango intercuartil de la serie temporal de datos nos permite identificar las irregularidades mencionadas. Dicho valor se ha fijado en 0.3 TECU/30s. De este modo se asume que las variaciones de TEC que superan este valor umbral son debidas a fluctuaciones de las señales producidas cuando éstas atraviesan las irregularidades de plasma ionosférico.

Las estructuras ionosféricas mencionadas pueden causar centelleo de amplitud y fase, llegando a alterar de manera notable la señal transmitida hasta el punto incluso de perder la señal y degradar así el posicionamiento (Kintner et al., 2007; Jakowski et al., 2008). Esto es debido, por un lado, al aumento en la dilución de precisión por razones geométricas (GDOP) y por otro, a la reestimación continuada de las ambigüedades. En este análisis se ha decidido mostrar el comportamiento de la pérdida de señal en los cuatro observables, el código y la fase en ambas frecuencias (L1 y L2), para analizar tanto su comportamiento temporal así como su influencia sobre el PPP. Se ha decidido diferenciar entre baja y alta elevación para destacar cuándo la causa puede ser puramente ionosférica o puede, por el contrario, deberse a obstáculos u otros fenómenos que no tengan que ver con la ionosfera. Para mantener coherencia con los umbrales anteriormente mencionados se ha utilizado el mismo umbral que en el cálculo del ROT, $45^{\circ}$. El número mínimo de épocas con pérdida de señal consideradas es de 3 (1'5 min) mientras que el máximo de 30 (15min). 
Tabla. 2. Receptores instalados en la estación KOUR. La primera columna muestra el periodo en el que el receptor estuvo operativo. Las siguientes tres columnas listan el tipo de receptor instalado, la serie de dicho receptor junto con su versión o actualización y la antena correspondiente. Por último se muestra el sistema GNSS con el que trabaja.

\begin{tabular}{|c|c|c|c|c|}
\hline Fecha & $\begin{array}{l}\text { Tipo de } \\
\text { receptor }\end{array}$ & Serie (firmware) & Antena (Num.) & $\begin{array}{c}\text { Sistema } \\
\text { GNSS }\end{array}$ \\
\hline $\begin{array}{l}\text { 01Ene- } \\
\text { 31Dic } \\
\mathbf{2 0 0 1}\end{array}$ & $\begin{array}{l}\text { Ashtech Z- } \\
\text { XII3 }\end{array}$ & $\begin{array}{c}\text { LP019995005 } \\
\text { (NAV CD00, } \\
\text { CHN 1D02*21) }\end{array}$ & AOAD/M_T (270) & GPS \\
\hline $\begin{array}{l}\text { 01Ene- } \\
\text { 30Sep } \\
\text { 30Sep- } \\
\text { 31Dic } \\
\mathbf{2 0 0 4}\end{array}$ & $\begin{array}{l}\text { Ashtech Z- } \\
\text { XII3 } \\
\text { Ashtech } \\
\text { UZ-12 }\end{array}$ & $\begin{array}{l}\text { LP019995005 } \\
\text { IR1200240020 } \\
\quad(\mathrm{CD} 00)\end{array}$ & $\begin{array}{c}\text { ASH701945C_M } \\
\text { (CR520014804) }\end{array}$ & GPS \\
\hline $\begin{array}{c}01- \\
06 \text { Ene }\end{array}$ & $\begin{array}{l}\text { Ashtech } \\
\text { UZ-12 }\end{array}$ & IR1200240020 & ASH701945C_M & GPS \\
\hline $\begin{array}{l}\text { 15Ene- } \\
08 \mathrm{Abr}\end{array}$ & $\begin{array}{c}\text { JPS } \\
\text { LEGACY }\end{array}$ & $340-0863(3.1)$ & $\begin{array}{c}\text { ASH701946.3 } \\
(\mathrm{CR} 420033512)\end{array}$ & GPS+GLO \\
\hline $\begin{array}{l}\text { 08Abr- } \\
\text { 13May }\end{array}$ & $\begin{array}{c}\text { JPS } \\
\text { LEGACY }\end{array}$ & $340-0863(3.2)$ & $\begin{array}{c}\text { ASH701946.3 } \\
\text { (CR420033512) }\end{array}$ & GPS+GLO \\
\hline $\begin{array}{l}\text { 13May- } \\
\text { 31Dic }\end{array}$ & $\begin{array}{c}\text { JPS } \\
\text { LEGACY }\end{array}$ & $340-0863(3.3)$ & $\begin{array}{c}\text { ASH701946.3 } \\
\text { (CR420033512) }\end{array}$ & GPS+GLO \\
\hline 2008 & & & & \\
\hline $\begin{array}{l}\text { 01Ene- } \\
\text { 31Dic }\end{array}$ & $\begin{array}{c}\text { JPS } \\
\text { LEGACY }\end{array}$ & $340-0863(3.4)$ & $\begin{array}{c}\text { ASH701946.3 } \\
(\mathrm{CR} 420033512)\end{array}$ & GPS+GLO \\
\hline 2011 & & & & \\
\hline
\end{tabular}

El posicionamiento se ha llevado a cabo en modo cinemático a través de la aplicación online CSRS-PPP proporcionada por el Natural Resources Canada, Canadian Spatial Reference System (Banville et al., 2009). El umbral en la elevación mínima de los satélites que se utiliza por defecto en dicho procesado es de $10^{\circ}$. Se ha decidido trabajar con la altitud estimada en el PPP debido a que la componente 
vertical es la más afectada por el empobrecimiento de la geometría de la constelación de satélites utilizados en el posicionamiento. Se ha calculado el error en la altura estimada, DH, mediante la diferencia entre el resultado proporcionado por CSRS-PPP y la posición IGS precisa de la estación. A partir del 14 de agosto del 2011, el servicio CSRS-PPP incluye por defecto observaciones de la constelación GLONASS (Global'naya Navigatsionnaya Sistema) ya que es el momento a partir del cual sus productos precisos están disponibles. Para remarcar los errores en el posicionamiento que puedan deberse a problemas derivados de las irregularidades se ha analizado el valor cuadrático medio (RMS) del error obtenido época por época en un intervalo en el que no se han observado fuertes variaciones de TEC (entre mayo y agosto de cada año) para definir un umbral, que se ha fijado en $30 \mathrm{~cm}$ para 2001 y 2004, y en $15 \mathrm{~cm}$ para 2008 y 2011. La diferencia de este valor umbral para cada grupo de datos es debida principalmente a la mejora producida en los receptores (tabla 2) y en los productos del IGS. En la figura 1 también se ha decidido mostrar los errores superiores a $50 \mathrm{~cm}$ para destacar las épocas de peor precisión.

\section{Resultados}

Debido a su localización, la estación KOUR sufre el efecto de las irregularidades de plasma ecuatorial en el primer y último cuatrimestre del año. En los gráficos se ha representado la falta de datos diarios con líneas horizontales blancas; cabe notar la ausencia de datos de Diciembre de 2001. En la primera columna de la figura 1 se presenta el comportamiento temporal del ROT superior a 0.3TECU/30s representando los resultados de cada año en una fila. Con respecto al comportamiento diario se ve claramente que las fluctuaciones rápidas del TEC se producen sobre todo tras la puesta de sol y hasta medianoche, llegando incluso a extenderse hasta varias horas después de la medianoche, sobre todo en periodos de moderada a alta actividad solar. En cuanto al comportamiento anual, como se ha mencionado, la aparición de las irregularidades se limita a los equinoccios pero está claramente desplazada hacia el solsticio de diciembre. Este comportamiento no es casual. Como ya han descrito diversos autores (Tsunoda, 1985; Gentile et al., 2011), las condiciones más propicias para la aparición de este tipo de fluctuaciones del contenido electrónico se producen en las épocas del año en las que el terminador solar se alinea con las líneas del campo magnético terrestre. De este modo, el gradiente del campo eléctrico zonal se hace máximo, y la deriva vertical debida al PRE es más acusada. Para la estación estudiada, dado que está situada en una zona en la que la declinación magnética es de unos $17^{\circ}$ hacia el Oeste, esta alineación se produce en las proximidades de los equinoccios pero tendiendo hacia el solsticio de invierno. Se puede ver que en épocas de baja actividad solar (año 2008), el porcentaje de casos con ROT superior a 1TECU/30s se reduce significativamente. Esta evidencia es debida en parte a que en los años de menor actividad solar la ionosfera presenta valores de densidad electrónica menores que en los de alta actividad y, por lo tanto, las diferencias que pueden existir entre las irregularidades y su entorno disminuye. Además, 
durante el primer cuatrimestre de 2008 también se ha reducido notablemente el número de épocas con ROT superior a 0.3 TECU/30s. Así mismo su aparición se ha retardado y la duración apenas sobrepasa la medianoche. Cabe aclarar que aunque en el análisis del ROT no se han considerado los satélites con elevación inferior a $45^{\circ}$, sí se ha observado que dichos satélites están igualmente afectados, en los mismos periodos de tiempo, por las fuertes variaciones del TEC.

Si observamos el error en la posición (segunda columna de la figura 1), podemos ver que, salvo en 2008, la aparición del error en la altitud estimada mediante PPP está estrechamente ligada al comportamiento del ROT, y aunque no en todas las épocas con fuerte ROT se observa un aumento de los errores por encima del valor umbral, éstos no son despreciables (véase tabla 3). El valor cuadrático medio de los errores es mayor en 2004 que en el resto de los años analizados. Esto puede ser debido entre otras causas a los problemas derivados de la geometría de la constelación que presenta abundantes épocas en las que se registran solo 5 satélites, el estado de los relojes, y otras causas no identificadas en este estudio. Estos errores se ven reflejados en la representación correspondiente a 2004 como alineaciones tanto diagonales como horizontales.

Tabla 3. Resultados obtenidos para cada año. En la segunda y tercera fila se presentan respectivamente, el porcentaje de épocas con valores del ROT por encima del valor umbral ( 0.3 TECU/30s) y el porcentaje de épocas en las que se registran errores en la altura estimada superiores a los umbrales ( $30 \mathrm{~cm}$ en 2001 y 2004 y $15 \mathrm{~cm}$ en 2008 y 2011); en la cuarta y quinta fila se muestra, el valor cuadrático medio del error en la altura estimada $\left(\mathrm{RMS}_{\mathrm{DH}}\right)$ en épocas de ROT inferior y superior al valor umbral .

\begin{tabular}{|c|c|c|c|c|}
\hline Intervalo de tiempo: 19:00-01:00LT & $\mathbf{2 0 0 1}$ & $\mathbf{2 0 0 4}$ & $\mathbf{2 0 0 8}$ & $\mathbf{2 0 1 1}$ \\
\hline \% épocas con ROT alto & 20.87 & 18.73 & 4.32 & 25.73 \\
\hline $\begin{array}{c}\text { \% épocas con errores superior al umbral } \\
\text { considerando las épocas de ROT alto }\end{array}$ & 17.52 & 8.33 & 1.77 & 13.22 \\
\hline RMS $_{\mathbf{D H}}$ considerando ROT bajo & 0.515 & 5.450 & 0.063 & 0.077 \\
\hline $\mathbf{R M S}_{\mathbf{D H}}$ considerando ROT alto & 1.470 & 45.76 & 0.056 & 0.308 \\
\hline
\end{tabular}

Tras observar que durante las épocas de altos valores de ROT aparecen numerosas pérdidas de la señal, se ha decidido comprobar cuáles son los observables que más a menudo se pierden. Cabría pensar que la frecuencia $\mathrm{f} 2$ es la más sensible a sufrir dichos problemas, ya que la potencia de esta señal es inferior a la de f1 y es más propensa a su debilitamiento debido a fenómenos como el centelleo; sin embargo se puede ver que su comportamiento, en este caso, dista de ser así (véanse las cuatro últimas columnas de la figura 1) y que además la pérdida de la señal depende 
claramente del tipo de receptor. En las figuras comentadas se puede distinguir que, por ejemplo, durante 2001 e inicios de 2004 los códigos de ambas frecuencias se pierden prácticamente en las mismas épocas. Los satélites de baja elevación sufren más pérdidas que los satélites de alta elevación en los periodos de ROT alto. Como ya se ha mencionado, aunque en las figuras del ROT solo se hayan mostrado los eventos correspondientes a satélites de alta elevación, los de baja elevación también registran fuertes fluctuaciones en las señales. En cuanto a lo que a las fases se refiere éstas prácticamente solo se pierden en satélites con elevación inferior a $45^{\circ}$.

En el año 2004 se ve que a partir de finales de mayo, el receptor empieza a sufrir mayores problemas en la recepción de la señal (en todos los observables) de algunos satélites. A finales de septiembre el receptor es reemplazado, pero no es hasta mediados de octubre cuando éste empieza a tener menos problemas. Tras este periodo se puede ver que hay un número abundante de satélites con baja elevación que se pierden, siendo los observables en f1 los que se pierden más frecuentemente. Sin embargo en épocas de ROT alto y para satélites con alta elevación, es la frecuencia f2 la que presenta mayores pérdidas.

Durante 2008 (época de baja actividad solar) las irregularidades observadas son de menor intensidad (los casos con ROT superior a 1TECU/30s han disminuido notablemente). También es posible apreciar que apenas se producen errores superiores a los $15 \mathrm{~cm}$ (en gris) y que la pérdida de señal observada no tiene que ver con la aparición de ROT elevado. Parece que, en general, durante este año las irregularidades observadas no son lo suficientemente fuertes como para producir problemas, como el centelleo de la señal, que deriven en la pérdida o deterioro de ésta, y que el receptor es lo suficientemente robusto como para recibirlas sin alteraciones que a posteriori produzcan una degradación en la precisión del posicionamiento. Sin embargo, se observa un aumento en la pérdida de señal de satélites con baja elevación desde mediados hasta finales de año, que no es tan acusado para L1 como lo es para el resto de observables.

Por último, en la cuarta fila se muestran los resultados correspondientes a 2011 (año de moderada y creciente actividad solar). Como cabría esperar, los errores más fuertes se producen en periodos en los que se han dado pérdidas de observables. Merece la pena mencionar la mayor robustez del receptor con respecto a L1 ya que, al contrario del resto de observables, no presenta un mayor número de pérdidas en los periodos en los que se observa un ROT alto.

Hay que hacer notar que las líneas diagonales que se observan en la figura 1 en las columnas correspondientes a la pérdida de señal, se deben en gran medida, a la obstrucción por elementos cercanos al suelo que alcanzan en general una elevación de $15^{\circ}$, pero que pueden llegar a $\operatorname{los} 30^{\circ}$ en ciertos años (como en 2008) y para acimuts concretos. 

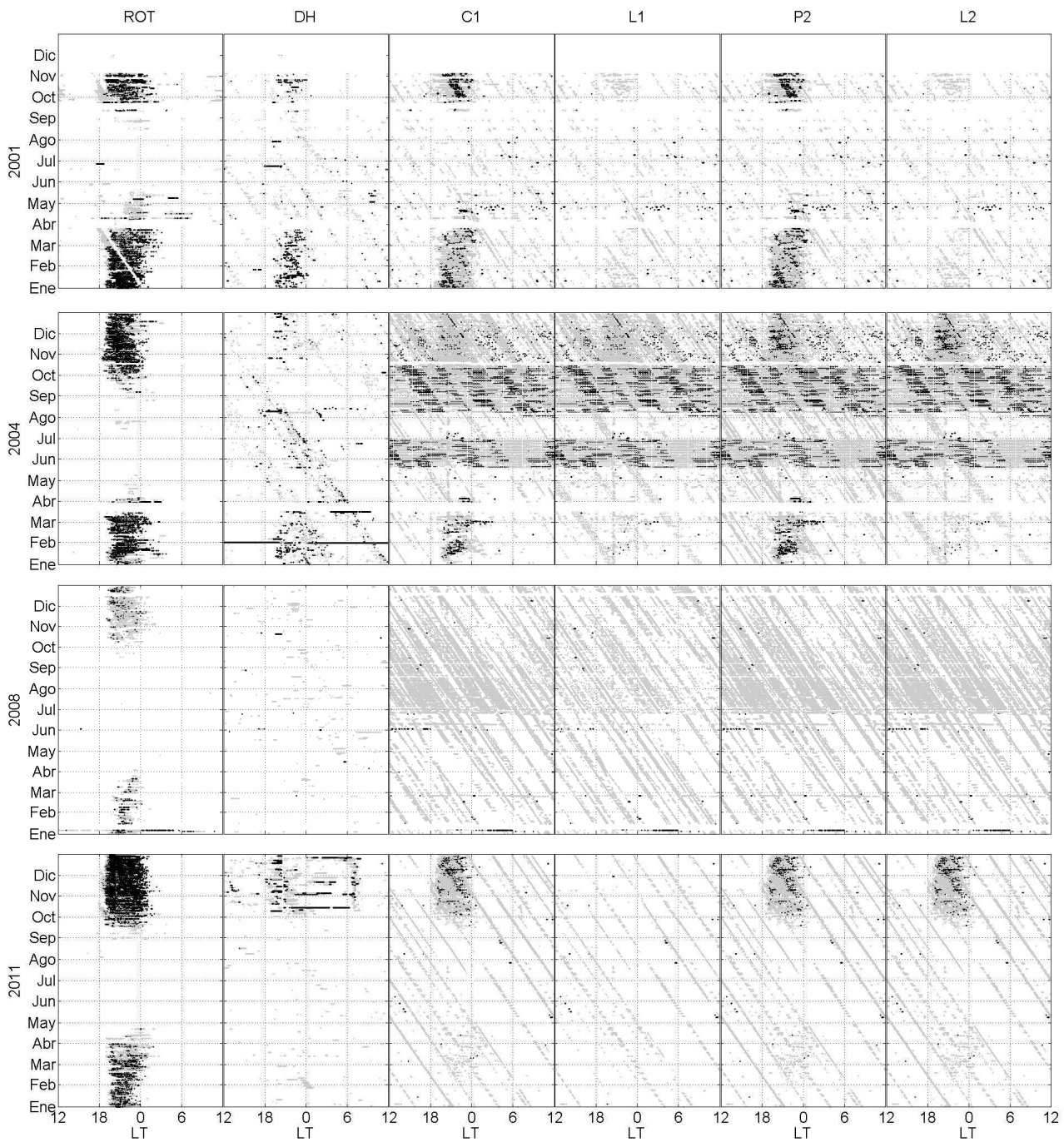

Fig. 1. Resultados para la estación de KOUR. Cada fila corresponde a un año. De arriba abajo: 2001, 2004, 2008 y 2011. De izquierda a derecha: ROT: en gris los valores por encima de 0.3 TECU/30s y en negro las épocas con valores superiores a 1 TECU/30s; DH (error en la altura estimada): en gris los valores superiores a $30 \mathrm{~cm}$ para 2001 y 2004 y los superiores a $15 \mathrm{~cm}$ para 2008 y 2011. En todos los casos los valores superiores a $50 \mathrm{~cm}$ en negro. Las últimas columnas representan la pérdida de los observables C1 (o P1), L1, P2 y L2 respectivamente: en gris los satélites con elevaciones comprendidas entre $10^{\circ}$ y $45^{\circ}$, y en negro, los satélites con elevación superior a $45^{\circ}$. 
En los casos analizados no solo se observa un gran número de pérdidas de señal sino que también se han detectado un gran número de saltos de ciclo en épocas con alto ROT (véase la figura 2). En esta figura también se observan (en 2001 y 2004) saltos de ciclo en patrones diarios (véanse las alineaciones horizontales de la figura 2 durante el año 2001 y 2004) que no tienen que ver con la aparición del ROT y no producen errores significativos en la altitud estimada.

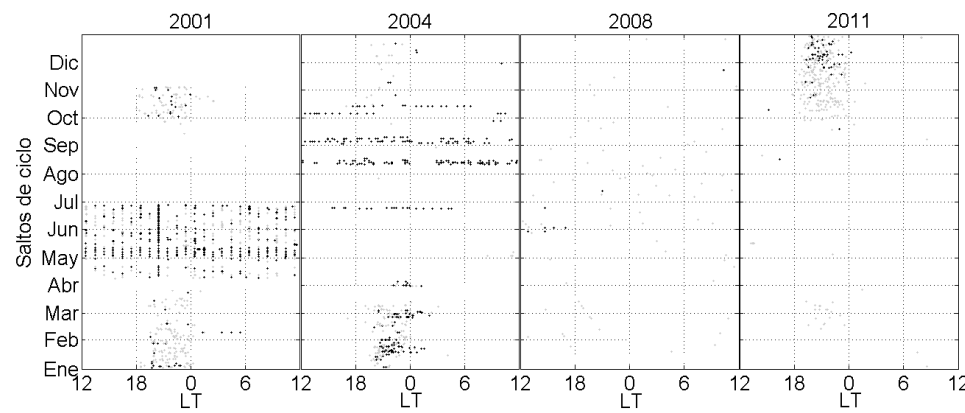

Fig. 2. Saltos de ciclo detectados en el preprocesado. De izquierda a derecha 2001, 2004, 2008 y 2011. En gris épocas en las que los saltos de ciclo detectados se producen en las señales de satélites con una elevación inferior a $45^{\circ}$ y en negro cuando la elevación es superior a ese valor.

\section{Conclusiones}

En este estudio se ha utilizado el ROT como indicador de la aparición de las irregularidades de plasma ecuatorial. Se ha observado que la manifestación de las fluctuaciones del contenido electrónico sobre KOUR presenta una fuerte dependencia temporal con mayor número de sucesos en las horas comprendidas entre la puesta del sol y la medianoche, en el primer y último cuatrimestre del año, con una disminución de sucesos a medida que la actividad solar decrece. También se ha mostrado que existe una clara relación entre las fluctuaciones del ROT debidas a irregularidades de plasma ionosférico y la degradación del posicionamiento puntual de precisión. El aumento del error en el posicionamiento sigue el comportamiento temporal observado en el ROT, debiéndose en gran medida, a la pérdida simultánea de los observables en épocas de fuertes fluctuaciones del contenido electrónico y a la sensibilidad del receptor para perder uno u otro observable. De modo que el aumento en la precisión observado con los años está relacionado con la capacidad del receptor para hacer el seguimiento de las señales sin que éstas se pierdan, resultando en una mayor capacidad posterior para llevar a cabo un posicionamiento de más precisión.

\section{Agradecimientos}

Este trabajo es parte de la actividad investigadora del "Grupo de Estudios Ionosféricos y Técnicas de posicionamiento Satelital (GNSS)" financiado por la Universidad 
Complutense de Madrid y la Comunidad de Madrid, y se ha llevado a cabo gracias al proyecto AYA2010-15501. I. Rodríguez-Bilbao agradece al Departamento de Educación, Política Lingüística y Cultura del Gobierno Vasco la concesión de la beca PREDOC. Los autores agradecen al Dr. Luigi Ciraolo el apoyo prestado y al Natural Resources of Canada la disponibilidad de su software para PPP así como a la comunidad IGS la accesibilidad a datos y productos GNSS. Los autores también quieren agradecer al revisor su ayuda en la evaluación de este trabajo.

\section{Referencias Bibliográficas}

ABDU, M.A. (2001). Outstanding problems in the equatorial ionospherethermosphere electrodynamics relevant to spread F. J. Atmos. Sol. Terr. Phys. 63, 869-884.

BANVILLE, S., Langley, R.B., Santos M.C. (2009). The precise point positioning software centre: an insight into online PPP service. Poster presented at the IAG 2009 meeting, Buenos Aires, Argentina, 31 Aug.

BASU, Sa., BASU, S., AARONS, J., McCLURE, J., COUSINS, M. (1978). On the coexistence of kilometer- and meter-scale irregularities in the nighttime equatorial F region. J. Geophys. Res. 83, A9, 4219-4226.

BISNATH, A., GAO, Y. Current state of precise point positioning and future prospects and limitations. In Proceedings of IUGG 24th General Assembly, 2007.

DE LACY, M.C., SANSÒ, F., GIL A.J., RODRÍGUEZ-CADEROT G. (2005). A method for the ionospheric delay estimation and interpolation in a local GPS network. Stud. Geophys. Geod. 49, 63-84.

DE LACY, M.C., GIL, A.J., RODRÍGUEZ-CADEROT, G., MORENO, B. (2008). A method to estimate the ionospheric bias by using the new GNSS frequencies: analysis of its theoretical accuracy in a PPP context. Física de la Tierra, 20, 133150.

GENTILE, L.C., BURKE, W.J., RODDY, P.A., RETTER, J.M., TSUNODA, R.T. (2011). Climatology of plasma density depletions observed by DMSP in the dawn sector. J. Geophys. Res. 116, A03321.

JAKOWSKI, N., MAYER, C., WILKEN, V., HOQUE M.M. (2008). Ionospheric impact on GNSS signals. Física de la Tierra, 20, 11-25.

KINTNER, P.M., LEDVINA B.M., DE PAULA E.R. (2007). GPS and ionospheric scintillation. Space Weather, 5, S09003.

LI, G., NING, B., LIU, L., REN, Z., LEI, J., SU, S.Y. (2007). The correlation of longitudinal/seasonal variations of evening equatorial pre-reversal drift and of plasma bubbles. Ann. Geophys. 25, 2571-2578.

MORENO, B., RADICELLA, S., DE LACY, M.C., HERRAIZ, M., RODRÍGUEZCADEROT, G. (2011). On the effects of the ionospheric disturbances on precise point positioning at equatorial latitudes. GPS Solutions, 15, 381-390. 
NAVA, B., RADICELLA, S.M., LEITINGER, R., COÏSSON, P. (2007). Use of total electron content data to analyze ionosphere electron density gradients. Adv. Space Res. 39,1292-1297.

RISBETH, H. (2000). The equatorial F-layer: progress and puzles. Ann. Geophys. $18,730-739$.

SCHAER, S. (1997). How to use CODE's Global Ionosphere Maps. Astronomical Institute, University of Bern.

SULTAN, P.J. (1996). Linear theory and modelling of Rayleigh-Taylor instability leading to the occurrence of equatorial spread F. J. Geophys. Res. 101, A12, 26875-26891.

TSUNODA, R.T. (1985). Control of the seasonal and longitudinal occurrence of equatorial scintillations by the longitude gradient in integrated $\mathrm{E}$ region Pedersen conductivity. J. Geophys. Res. 90, A1, 447-456.

WHALEN, J.A. (2002). Dependence of equatorial bubbles and bottomside spread F on season, magnetic activity, and ExB drift velocity during solar maximum. $J$. Geophys. Res. 107, A2, 1024. 